\title{
The Crab pulsar at VHE
}

\author{
Roberta Zanin ${ }^{1, a}$ \\ ${ }^{1}$ Max-Planck-Institut fur Kernphysik, P.O. Box 103980, D 69029 Heidelberg, Germany
}

\begin{abstract}
.
The last six years have witnessed major revisions of our knowledge about the Crab Pulsar. The consensus scenario for the origin of the high-energy pulsed emission has been challenged with the discovery of a very-high-energy power law tail extending up to $\sim 400$ $\mathrm{GeV}$, above the expected spectral cut off at a few GeV. Now, new measurements obtained by the MAGIC collaboration extend the energy spectrum of the Crab Pulsar even further, on the $\mathrm{TeV}$ regime. Above $\sim 400 \mathrm{GeV}$ the pulsed emission comes mainly from the interpulse, which becomes more prominent with energy due to a harder spectral index. These findings require $\gamma$-ray production via inverse Compton scattering close to or beyond the light cylinder radius by an underlying particle population with Lorentz factors greater than $5 \times 10^{6}$. We will present those new results and discuss the implications in our current knowledge concerning pulsar environments.
\end{abstract}

\section{Introduction}

Pulsars are rapidly rotating neutron stars (NS) which form highly magnetized magnetospheres, usually described as magnetic dipoles. The magnetosphere is filled with charged particles, mainly electrons and positrons, extracted from the stellar surface by the induced electric fields. The original model for pulsar magnetospheres foresees that these charged particles satisfy the ideal magnetohydrodynamic (MHD) $(E \cdot B=0)$ and the free-force (FF, $\rho E+J \times B=0)$ conditions [1]. In this view, the magnetic field lines cannot remained closed beyond the light cylinder ( $\mathrm{LC}$, with a radius $\mathrm{R}_{L C}$ ), where the angular velocity of the NS is equal to the speed of light. Along the open field lines the plasma flows along asymptotically monopole field lines [2] forming the pulsar wind region. Nowadays it is commonly accepted that the ideal MHD and FF conditions cannot be fulfilled everywhere inside the magnetosphere. In the past years regions where the electric field is not totally screened by the plasma were invoked as possible sites of particle accelerations. They are referred as to gaps. Recently current sheets are taking over though. They are current carrying surfaces where particles can be accelerated via relativistic reconnection and radiate synchrotron emission from optical to $\gamma$-ray wavelengths. In particular, an equatorial current sheet was proposed by Contopoulos to ensure the closure of the largescale currents flowing from the star to infinity and back [3], but only recent particle-in-cell (PIC) simulations of plasma-filled magnetospheres have provided the evidence for its existence, as well as its possible extension down to the NS surface, separating the open and closed field lines (this names them separatrix) [4-7]. The equatorial current sheet separates the field lines that originate from the

\footnotetext{
a e-mail: Roberta.Zanin@mpi-hd.mpg.de
} 
two pulsar magnetic poles. When the rotation and magnetic axes are not aligned (oblique pulsars), this current sheet develops corrugation whose amplitude increases linearly with the distance from the star. The equatorial plan is then divided into stripes of magnetic field of opposite polarities, hence the name stripped wind [8-10].

In this new paradigma of the pulsar magnetosphere, two classes of pulsars are defined depending on the particle density inside the magnetosphere: the high-multiplicity pulsars where pair production takes place within the magnetosphere, that are the young pulsars with high stellar magnetic fields, and the low-density pulsars where particles are supplied only from the NS surface and are identified with the old pulsars. For the first class of pulsars the FF solution is a good approximation of their magnetosphere characterized by the presence of the separatrices and the equatorial current sheet. They are weakly dissipative $[7,11]$. The low-density pulsars exhibit a charged separated magnetosphere with no separatrices since the equatorial sheet is electrostatically supported. They show high dissipation (>40\%) beyond the LC [7, 11].

Pulsars are observed from radio frequencies up to $\gamma$ rays. Radio emission is believed to be produced at low-altitudes, close to the stellar magnetic poles, by a coherent process either maser amplification or coordinated motion of group of charges. At higher frequencies pulsed emission is incoherent, most likely synchrotron radiation produced by relativistic electron-positron pairs. At high energies, pulsed emission was long attributed to synchro-curvature by particles accelerated in magnetospheric gaps. According to the location of the gap inside the magnetosphere, $\gamma$-ray pulsar models were classified into polar caps, if close to the magnetic poles, [12], outer gaps if at high altitudes [13], or slot gaps, if along the last open field lines [14]. In particular, such a synchro-curvature emission has a maximum energy limited by radiation losses or magnetic and $\gamma-\gamma$ pair absorption. The recent advances on the high-energy pulsar physics due to discovery of more than $150 \gamma$-ray pulsars by Fermi/LAT [15] suggest that pulsed $\gamma$ rays are produced at high altitudes favouring the outer gaps models. This conclusion is mainly drawn by two observational facts: 1) The spectral cutoff at a few $\mathrm{GeV}$ is exponential, and often even subexponetial for young pulsars. The smoother spectral break is interpreted as the overlapping of emission beams coming from different regions and/or with different cutoff energies. 2) The majority of the $\gamma$-ray pulsars present a double-peaked pulsar profile, which in low-altitude emission models would require a specific geometric configuration (both viewing and inclination angles close to $90^{\circ}$ ), too special to meet the observed numbers [16]. However, in the context of the new paradigma of the pulsar magnetosphere, the high-energy emission could entirely or partially be of synchrotron origin produced in the equatorial current sheet beyond the light cylinder $[17,18,26]$.

\section{The Crab pulsar}

The Crab pulsar is the second most powerful pulsar known so far with a spin-down luminosity $\dot{E}=$ $3.8 \times 10^{38} \mathrm{erg} / \mathrm{s}$ and the only pulsar whose age is known with precision. It is, in fact, the leftover of an historical supernova explosion occurred in $1054 \mathrm{AD}$ which was in detail documented by Chinese astronomers.

Its pulse profile is characterised by two peaks almost aligned at almost all wavelengths, even though their amplitudes change with energy $[19,20]$. The main peak at low radio frequencies $(0.1-5$ $\mathrm{GHz}$ ), by definition set at phase 0 , is usually referred to as $\mathrm{P} 1$, whereas $\mathrm{P} 2$ or interpulse is located $\sim 0.4^{\circ}$ away. The emission in between the two peak is called bridge. 


\subsection{Recent results at VHE}

All the $160 \gamma$-ray pulsars detected by Fermi/LAT show a spectral break at $\sim$ few $\mathrm{GeV}$ in agreement with the synchro-curvature scenario [15], but one: the Crab pulsar. The spectrum of the Crab pulsar between $0.1-100 \mathrm{GeV}$ is well parametrized by a power-law function with a sub-exponential cutoff $(b<1)$. The best-fit value for the photon index is $\gamma=1.59 \pm 0.01$, for the energy break is $(5.09 \pm 0.63) \mathrm{GeV}$ and for the curvature index $b=0.43 \pm 0.01$ [21]. Recent measurements of the Crab pulsar by imaging atmospheric Cherenkov telescopes showed a totally unexpected power-law component emerging above the synchro-curvature cutoff and extending above $100 \mathrm{GeV}$ [22-24]. This new spectral component, that is excluding the spectral break at more than than $6 \sigma$ [22], is a common feature of both peaks and the bridge. A joint fit of the Fermi/LAT data above $10 \mathrm{GeV}$ and the MAGIC ones indicates that $\mathrm{P} 2$ is significantly harder than $\mathrm{P} 1$ with a difference between the photon indices of $0.5 \pm 0.1$. Whereas the steeper P1 $(\Gamma=3.5 \pm 0.1)$ spectrum cannot be detected beyond $600 \mathrm{GeV}, \mathrm{P} 2$ becomes the dominant component above $50 \mathrm{GeV}$ [23] and extends up to $1.5 \mathrm{TeV}$ without any sign of cutoff [25]. A lower limit on the spectral cutoff is estimated at $700 \mathrm{GeV}$. On the other hand, the spectrum of the bridge is as soft as P1 and fades out already at $150 \mathrm{GeV}$ [20].

This VHE pulsed emission is very unlikely to be synchro-curvature radiation: the production of $1 \mathrm{TeV}$ photon would require, in fact, a curvature radius of the magnetic field lines one order of magnitude larger than the usual adopted one. If emission up to few hundreds of $\mathrm{GeV}$ could be also synchrotron radiation from the equatorial current sheet $[17,26]$, the emission at higher energies is accredited to be produced via inverse Compton scattering. In this view several models have been put forward. Some of them are listed in the following:

- inverse Compton scattering of relativistic wind electrons off pulsed optical/X-ray photons with magnetospheric origin $[27,28]$. This model was proposed to explain the emission up to $400 \mathrm{GeV}$ and can well reproduce the pulse profile by assuming an anisotropic pulsar wind. Nevertheless, it fails in reproducing the spectrum up to $1 \mathrm{TeV}$. The production of these energetic photons would require electron parent population with a Lorentz factor larger than $5 \times 10^{6}$, hence a continuos acceleration from the $\mathrm{LC}$ up to $\sim 100 \mathrm{R}_{L C}$ which in turn results in an overestimation of the $\mathrm{GeV}$ flux.

- synchrotron-self-Compton scattering off synchrotron photons produced in the current sheet by the same population of synchrotron-emitting electrons. This model reproduces the spectrum up to VHE as a sum of two distinct components: the synchrotron power-law up to few hundreds of $\mathrm{GeV}$ and the SSC as an extra bump fading out at a few $\mathrm{TeV}$ [17]. However, the model cannot explain the pulse profile.

- inverse Compton scattering off either photons in particle cascading inside the magnetospheric gaps $[23,29,30]$.

So far none of the existing model can explain simultaneously the spectrum and the pulse profile with its narrow peaks at VHE. In addition there is no comprehensive theoretical model reproducing all the observable of the pulsed emission i.e. the broadband spectrum with its spectral changes across the pulse-phase in $\mathrm{X}$ - and $\gamma$ rays, and the pulse profile with its energy-dependent peak amplitude and width.

\section{References}

[1] P. Goldreich, W.H. Julian, ApJ 157, 869 (1969)

[2] F.C. Michel, ApJ 180, 207 (1973)

[3] I. Contopoulos, D. Kazanas, C. Fendt, ApJ 511, 351 (1999) 


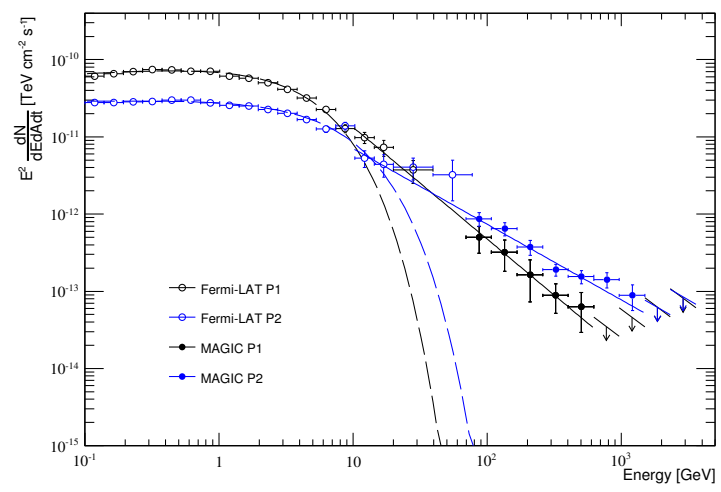

Figure 1. Phase-folded SED of the Crab P1 (black circles) and P2 (blue circles) at HE and VHE (open and filled circles). Taken from [25].

[4] A. Spitkovsky, ApJ, Letters 648, L51 (2006)

[5] C. Kalapotharakos, I. Contopoulos, $A$ E A 496, 495 (2009)

[6] A. Tchekhovskoy, A. Spitkovsky, J.G. Li, MNRAS 435, L1 (2013)

[7] B. Cerutti, A. Philippov, K. Parfrey, A. Spitkovsky, MNRAS 448, 606 (2015)

[8] F.V. Coroniti, ApJ 349, 538 (1990)

[9] S.V. Bogovalov, AAP 349, 1017 (1999)

[10] J.G. Kirk, O. Skjæraasen, Y.A. Gallant, AAP 388, L29 (2002)

[11] I. Contopoulos, MNRAS 463, L94 (2016)

[12] A.K. Harding, E. Tademaru, L.W. Esposito, ApJ 225, 226 (1978)

[13] K.S. Cheng, C. Ho, M. Ruderman, ApJ 300, 500 (1986)

[14] A.G. Muslimov, A.K. Harding, ApJ 588, 430 (2003), astro-ph/0301023

[15] F. collaboration, $A p J$ 208, 17 (2013)

[16] R.W. Romani, K.P. Watters, ApJ 714, 810 (2010)

[17] I. Mochol, J. Pétri, MNRAS 449, L51 (2015)

[18] B. Cerutti, A.A. Philippov, A. Spitkovsky, MNRAS 457, 2401 (2016)

[19] L. Kuiper, et al, $A \mathcal{E} A \mathbf{3 7 8}, 918$ (2001)

[20] MAGIC Collaboration, $A \mathcal{E} A$ 565, L12 (2014)

[21] R. Buehler, et al., $A p J$ 749, 26 (2012)

[22] VERITAS Collaboration, Science 334, 69 (2011)

[23] MAGIC Collaboration, ApJ 742, 43 (2011)

[24] MAGIC Collaboration, $A \mathcal{E} A$ 540, A69 (2012)

[25] MAGIC Collaboration, $A \mathcal{E} A$ 585, A133 (2016)

[26] J. Pétri, MNRAS 424, 2023 (2012)

[27] S.V. Bogovalov, F.A. Aharonian, MNRAS 313, 504 (2000)

[28] F.A. Aharonian, S.V. Bogovalov, D. Khangulyan, Nature 482, 507 (2012)

[29] M. Lyutikov, N. Otte, A. McCann, ApJ 754, 33 (2012)

[30] K. Hirotani, ApJ, Letters 798, L40 (2015) 